\title{
Nanometer-size anisotropy of injection-molded polymer micro-cantilever arrays
}

\author{
Prabitha Urwyler, ${ }^{1,2}$ Hans Deyhle, ${ }^{1,3}$ Oliver Bunk, ${ }^{3}$ Per Magnus Kristiansen, ${ }^{4}$ \\ and Bert Müller ${ }^{1, a)}$ \\ ${ }^{1}$ Biomaterials Science Center, University of Basel, clo University Hospital, 4031 Basel, Switzerland \\ ${ }^{2}$ Laboratory for Micro- and Nanotechnology, Paul Scherrer Institute, 5232 Villigen PSI, Switzerland \\ ${ }^{3}$ Swiss Light Source, Paul Scherrer Institute, 5232 Villigen PSI, Switzerland \\ ${ }^{4}$ Institute of Polymer Engineering (IKT) and Institute of Polymer Nanotechnology (INKA), \\ School of Engineering, University of Applied Sciences and Arts Northwestern Switzerland, 5210 Windisch, \\ Switzerland
}

(Received 5 January 2012; accepted 23 April 2012; published online 30 May 2012)

\begin{abstract}
Understanding and controlling the structural anisotropies of injection-molded polymers is vital for designing products such as cantilever-based sensors. Such micro-cantilevers are considered as cost-effective alternatives to single-crystalline silicon-based sensors. In order to achieve similar sensing characteristics, structure and morphology have to be controlled by means of processing parameters including mold temperature and injection speed. Synchrotron radiation-based scanning small- (SAXS) and wide-angle X-ray scattering techniques were used to quantify crystallinity and anisotropy in polymer micro-cantilevers with micrometer resolution in real space. SAXS measurements confirmed the lamellar nature of the injection-molded semi-crystalline microcantilevers. The homogenous cantilever material exhibits a lamellar periodicity increasing with mold temperature but not with injection speed. We demonstrate that micro-cantilevers made of semi-crystalline polymers such as polyvinylidenefluoride, polyoxymethylene, and polypropylene show the expected strong degree of anisotropy along the injection direction. (C) 2012 American Institute of Physics. [http://dx.doi.org/10.1063/1.4720942]
\end{abstract}

\section{INTRODUCTION}

Injection-molded polymer parts exhibit a skin-core morphology and the related mechanical properties. They naturally show a relatively sharp transition between the homogeneous core with spherulite crystallites and the inhomogeneous skin composed of oriented and elongated crystallites, referred to as the shish-kebab structure. ${ }^{1}$

The injected polymer melt cools down at the mold wall within milliseconds via the heat conducting metal (Fig. 1), which leads to the formation of an oriented but amorphous skin layer, here termed Zone A. ${ }^{2}$ Adjacent to this skin layer, the highly oriented transient layer, termed Zone B, forms. Since Zone A acts as a heat flow barrier, partial crystallization in Zone B takes place. As the heat transfer from the polymer toward the mold becomes less and less efficient, the intermediate shear layer, Zone C, develops. It is distinguished by the partial relaxation of shear-induced orientations before solidification. ${ }^{1-4}$ After cessation of the polymer flow, the cooling rate in the core, termed Zone D, is so low that only spherulitic superstructures ${ }^{2}$ with relaxed chains are established. The thickness of the four zones strongly depends on the processing and can also be manipulated incorporating nucleating agents. ${ }^{5,6}$ The mold temperature affects the solidification of the selected polymer. ${ }^{7}$ The fraction of the skin layer markedly increases with melt temperature, while the fraction of the shear zone varies with the injection pressure. ${ }^{8}$

\footnotetext{
${ }^{a)}$ E-mail: bert.mueller@unibas.ch. Tel.: +41 (0)61 26596 60. Fax: +41 (0)612659699.
}

The skin-core morphology not only depends on the process parameters but also on the shape and size of the polymer products. It has been pointed out that higher molecular orientation occurs with decreasing cavity thickness. ${ }^{9}$ Therefore, we expect to locate highly ordered polymer molecules in injection-molded micro-cantilevers.

The correlation between structure and function is known from the literature. ${ }^{5}$ The mechanical properties of the injection-molded polymer products significantly derive from the highly oriented skin layers formed through shear-induced crystallization. ${ }^{5}$ The skin layer may become dominant in microstructures. For micro-cantilevers $(\mu \mathrm{C})$, the skin might even fill the entire cross-section, so that the isotropic core is absent. Therefore, the bending characteristics of $\mu \mathrm{Cs}$ may crucially depend on the selected process parameters including mold temperature and injection pressure.

The understanding of the $\mu \mathrm{C}$ properties requires detailed analysis of their structure including anisotropy. The present communication concentrates on spatially resolved small- and wide-angle synchrotron $\mathrm{x}$-ray scattering (SAXS and WAXS) as these methods cover the entire nanometer range. ${ }^{10}$ Both techniques were already used to reveal a gradual change of molecular orientation from the periphery to the center of injection-molded specimens. ${ }^{11,12}$

It should be noted that amorphous and semi-crystalline polymers have been used for injection molding of polymeric micro-cantilevers. ${ }^{13}$ Amorphous polymers show homogeneous arrangement of molecules and a lack of short- and longrange orders. Semi-crystalline polymers generally exhibit ordering on molecular and supra-molecular levels. The semi- 


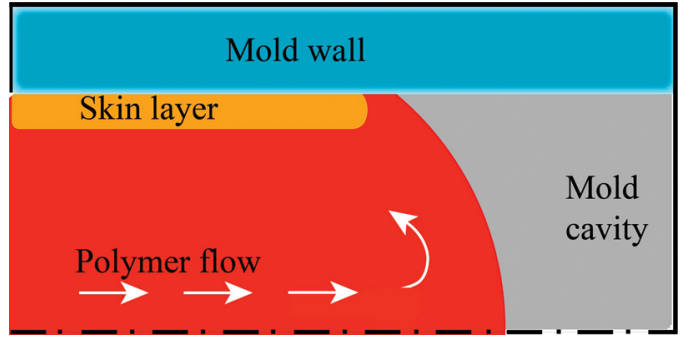

FIG. 1. Mold filling involves flow of polymer melts and solidification of the melt starting at the walls. Fountain flow describes how the polymer fills the mold cavity. Molecules from the center of the cavity flow towards the wall and form a stable skin layer. This causes a higher degree of molecular orientation in the skin layer compared to bulk.

crystalline polymers represent a characteristic lamellar morphology of stacks composed of crystalline and amorphous regions. These stacks contain crystals of distinctive thickness $\left(I_{c}\right)$ and amorphous layers of thickness $I_{\mathrm{a}}$ in between. These lengths define the long period as $L=I_{c}+I_{\mathrm{a}}$ which is in the order of $10 \mathrm{~nm}$ and depends on the selected polymer. ${ }^{14}$

\section{EXPERIMENTAL}

\section{A. Injection molding}

A laser-machined mold insert was used for the fabrication of arrays of $8 \mu \mathrm{C}$, featuring cavities of $480 \mu \mathrm{m} \times 100 \mu \mathrm{m}$ $\times 45 \mu \mathrm{m}$. The first generation of the mold insert (Steel 1.2312) was micro machined using a ns-laser (YAG, Coeheret Avia, wavelength $355 \mathrm{~nm}$, pulse duration $20 \mathrm{~ns}$ ). The secondgeneration mold insert incorporated high-quality steel with small grain size (Polmax Uddeholm) and was micro machined using a ps-laser ablation system (YAG-laser, SuperRapid, Lumera, $355 \mathrm{~nm}$, pulse duration $10 \mathrm{ps}$ ). The small grain size of the mold material along with ps-laser ablation resulted in sharp edges, better accuracy, and less roughness. Injectionmolding experiments were performed on an Arburg 320 ALLROUNDER (Arburg, Lossburg, Germany).

The following polymer materials were injection molded at the given temperatures of melt $T_{\text {melt }}$, polyetheretherketone (PEEK: AvaSpire AV-650 BG15, Solvay Advanced Polymers $\mathrm{GmbH}$, Düsseldorf, Germany, $T_{\text {melt }}=400^{\circ} \mathrm{C}$ ), polypropylene (PP: Metocene HM 648 T, LyondellBasell, Bayreuth, Germany, $T_{\text {melt }}=200^{\circ} \mathrm{C}$ ), polyoxymethylene copolymer (POM: $511 \mathrm{P}$ Delrin NC010, $T_{\text {melt }}=220^{\circ} \mathrm{C}$ ), cyclic olefin copolymer (COC: Topas 8007 X 10, TOPAS Advanced Polymers $\mathrm{GmbH}$, Frankfurt-Höchst, Germany, $T_{\text {melt }}=240^{\circ} \mathrm{C}$ ), and polyvinylidenefluoride (PVDF: Kynar 720, Arkema Puteaux, France, $T_{\text {melt }}=220^{\circ} \mathrm{C}$ ). Details on the commercial polymer grades used as well as supplementary information on the processing are published. ${ }^{13}$ For this study, the polymer $\mu \mathrm{C}$ were injection molded at the two following mold temperatures ( $\left.T_{\text {mold }}\right)$ keeping the injection speed at $9 \mathrm{~cm}^{3} / \mathrm{s}\left(\mathrm{PP}: 40^{\circ} \mathrm{C}\right.$, $80^{\circ} \mathrm{C}$; PVDF: $80^{\circ} \mathrm{C}, 120^{\circ} \mathrm{C}$; POM: $120^{\circ} \mathrm{C}, 150^{\circ} \mathrm{C}$ ). The dependence of the structure on injection speed for PP 5, 10, and $20 \mathrm{~cm}^{3} / \mathrm{s}$; for PVDF 10 and $20 \mathrm{~cm}^{3} / \mathrm{s}$; and for POM 10 and $20 \mathrm{~cm}^{3} / \mathrm{s}$ was studied as well.

The micro-cantilevers were produced in batches of 20 arrays. One array per batch was investigated using $\mathrm{x}$-ray scattering.

\section{B. X-ray scattering}

The $8 \mu \mathrm{C}$ of each individual array were examined using scanning SAXS and WAXS. SAXS and WAXS patterns of the injection-molded micro-cantilever arrays were obtained at the cSAXS beamline at the Swiss Light Source, Villigen, Switzerland, ${ }^{10}$ at a photon energy of $11.2 \mathrm{keV}$ (wavelength $1.107 \AA$ ). SAXS data were acquired within three distinct beamtimes. The $\mathrm{x}$-ray beam was focused to about $25 \mu \mathrm{m} \times 5 \mu \mathrm{m}, \quad 30 \mu \mathrm{m} \times 30 \mu \mathrm{m}$, and $25 \mu \mathrm{m} \times 5 \mu \mathrm{m}$ in horizontal and vertical directions, respectively. Silver behenate powder diffraction served to determine the sample-detector distance of $7.1 \mathrm{~m}$ for SAXS and $0.4 \mathrm{~m}$ for WAXS measurements.

The micro-cantilever arrays were mounted on a frame and translated perpendicular to the beam by means of a motorized 2D manipulator. Diffraction patterns were recorded on a 2D PILATUS detector with a pixel size of $172 \mu \mathrm{m} .{ }^{10}$ The exposure time was $2 \mathrm{~s}$ for WAXS and $0.150 \mathrm{~s}$ for SAXS. For WAXS, the specimens were mounted with the cantilevers oriented vertically. For SAXS, the specimens were mounted with the cantilevers oriented horizontally to study the cantilever rim region with highest possible resolution. The scanning was performed line wise: the specimens were moved in the horizontal direction through the beam at constant speed while the $\mathrm{x}$-ray detector recorded data continuously. The covered length on the specimen for each frame corresponded to 20, 25, and $15 \mu \mathrm{m}$, respectively, while the distance between the lines corresponded to 10,20 , and $15 \mu \mathrm{m}$. The air scattering was collected for a sample-free area and was subtracted from each frame. Data treatment was performed using selfwritten MATLAB 7.6.0 (The MathWorks, Inc., MA, USA) code. $^{10}$

The arrays for the temperature-dependent study were measured within two beamtimes with step sizes of $20 \mu \mathrm{m} \times 10 \mu \mathrm{m}$ and $20 \mu \mathrm{m} \times 25 \mu \mathrm{m}$. This means, $48 \mu \mathrm{C}$ per material were examined. The arrays for the speed-dependent study were examined during another beamtime with a step size of $15 \mu \mathrm{m} \times 15 \mu \mathrm{m}$. Here, $48 \mu \mathrm{C}$ for PP, $24 \mu \mathrm{C}$ for PVDF, and $24 \mu \mathrm{C}$ for POM were included into the study.

\section{RESULTS AND DISCUSSION}

\section{A. Injection molding}

Fig. 2 shows images of injection-molded polymeric $\mu \mathrm{C}$ using two generations of molds. The improved mold fabrication led to a much better shape of the $\mu \mathrm{C}$ (cf. images in the first and the second row in Fig. 2). The polymers PP, POM, and PVDF completely filled the mold cavities in spite of the high-aspect ratio of the microstructure (cf. images for PP in Fig. 2). PEEK, which requires mold temperatures above $320^{\circ} \mathrm{C}$, only partially filled the mold. For the COC $\mu \mathrm{C}$, successful de-molding was usually impossible owing to its inherent brittleness.

The two optical micrographs on the left side of Fig. 2 display dark rim-like regions at the edges of each $\mu \mathrm{C}$. These rim-like regions might be the result of slant edges causing refraction. 

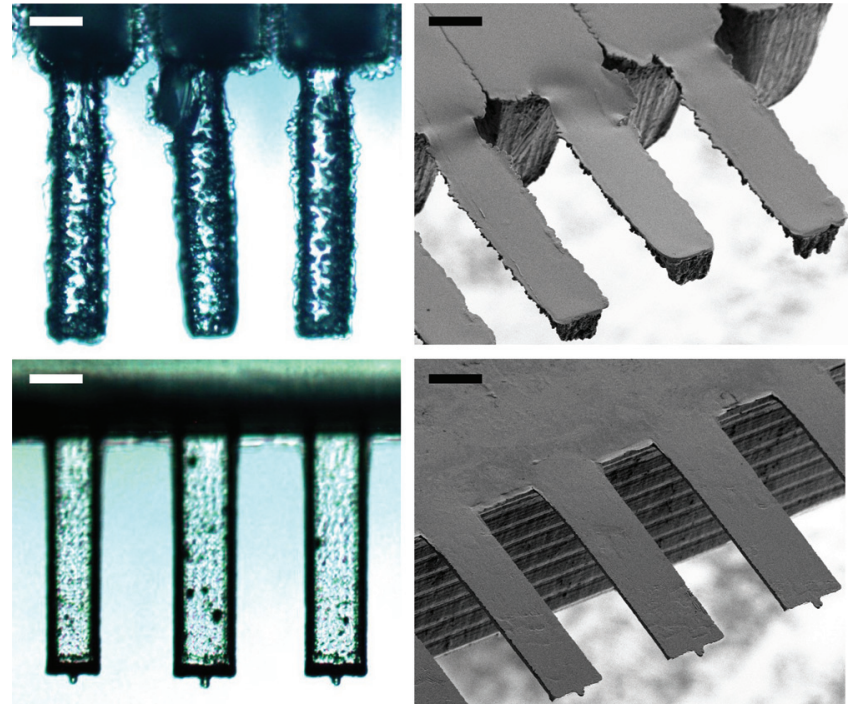

FIG. 2. Optical and SEM micrographs of injection-molded PP $\mu \mathrm{C}$ (mold temperature $80^{\circ} \mathrm{C}$ ). The scale bars correspond to $100 \mu \mathrm{m}$. The images of the first and second rows demonstrate the development from the first- to the second-generation injection molds. The optical micrographs show rim-like regions at the edges of the $\mu \mathrm{C}$.

\section{B. Wide-angle $x$-ray scattering}

WAXS supports the identification of crystalline phases, the degree of crystallinity, and the average orientation of molecules. Crystalline materials give rise to diffraction patterns with spots and/or sharp rings, whereas amorphous materials only produce broad, diffuse rings characteristic for the short-range order.

Fig. 3 represents 2D WAXS patterns of the four polymers COC, PEEK, POM, and PP measured at the rim regions and the center of the $\mu \mathrm{C}$. The patterns from rim and center only differ in intensity. This difference results from the $\mu \mathrm{C}$ 's thicknesses, which is smaller at the $\mu \mathrm{C}$ rim. Note that the two distinctive spots present in all images of Fig. 3 are artifacts, i.e., diffraction from the x-ray beam exit window made of mica muscovite, a single crystalline mineral.

WAXS patterns from COC and PEEK $\mu \mathrm{C}$ exhibit a halo, which is characteristic for amorphous materials without

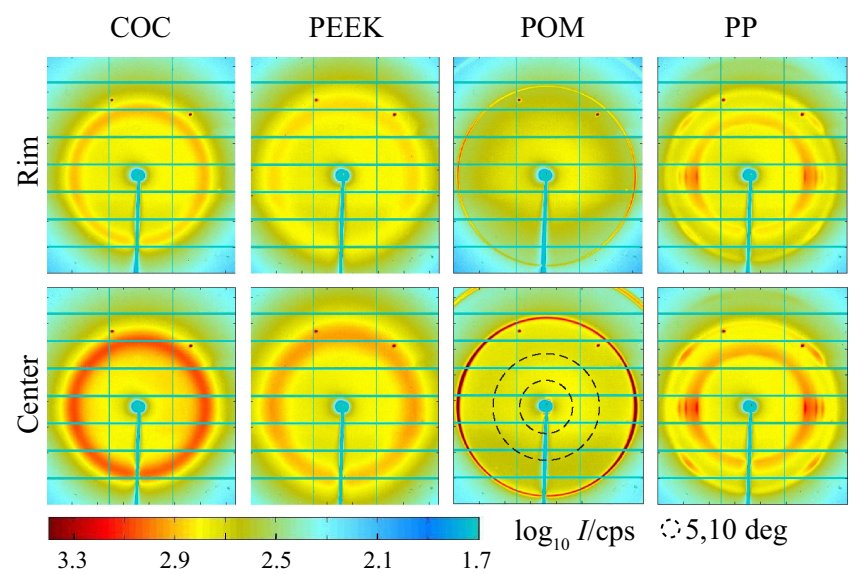

FIG. 3. Wide-angle $\mathrm{x}$-ray scattering patterns of the first generation $\mu \mathrm{C}$ measured at the rim (top row) and center (bottom row). The WAXS patterns from rim and center only differ in intensity. preferred orientation. For COC, the co-polymerization of ethylene with norbornene renders the structure amorphous. PEEK, which is generally found in a semi-crystalline state, was found to be amorphous. We assume that this is due to the fast cooling rate during $\mu \mathrm{C}$ fabrication.

The WAXS patterns of POM and PP show features characteristic for semi-crystalline structures with moderate (POM) or even quite distinct (PP) average orientation of molecules. For POM, a sharp ring at $q=16.21 \mathrm{~nm}^{-1}$ is present, which corresponds to a spacing of $d=0.38 \mathrm{~nm}$. Note that the two dashed circles represent scattering angles of $5^{\circ}$ and $10^{\circ}$, respectively. The radial integration of the WAXS patterns from PP reveals the presence of the distinct peaks at $q$-values of $15.45,13.16,12.02,10.66$, and $10.12 \mathrm{~nm}^{-1}$. The related $d$-values, i.e., $0.40,0.47,0.52,0.58$, and $0.62 \mathrm{~nm}$, correspond to the $\alpha$ and $\beta$ phases of PP.

\section{Small angle $x$-ray scattering}

The optical micrographs of Fig. 2 show a rim region around each $\mu \mathrm{C}$, which is also visible in the spatially resolved SAXS patterns (see inset of Fig. 4). The colorcoded SAXS data of the inset illustrate the preferential orientation of the scattering, which is found to be perpendicular to the $\mu \mathrm{C}$ near the edges (cf. color wheel in Fig. 4). The integral scattered intensity across individual COC, PP, POM, and PEEK $\mu \mathrm{C}$ reveals strong edge signals, which are much stronger, compared to the signal from the $\mu \mathrm{C}$ 's center and can be fitted by means of Gaussians. The full-width-at-halfmaximum (FWHM) of the Gaussians does not depend on the selected polymer and amounts to $(21.8 \pm 0.5) \mu \mathrm{m}$. From our point of view, it is implausible that this width, constant for the four selected polymer materials, relates to the skin layer thickness. Therefore, we assume that the presence of these strong edge signals arises from edge scattering of the elliptically shaped $\mathrm{x}$-ray beam within the sloped region of the

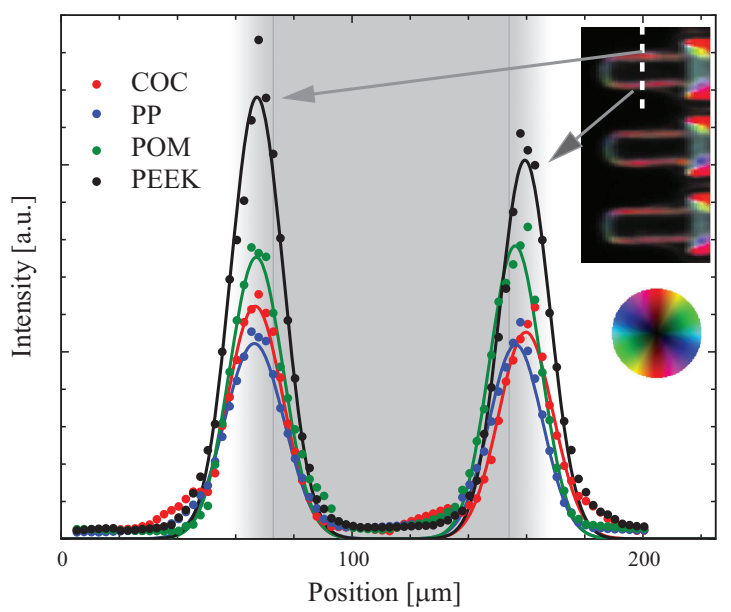

FIG. 4. SAXS intensity distribution $(286-418 \mathrm{~nm})$ integrated along the first generation $\mu \mathrm{C}$. High scattering intensities are present at the rim-like regions for COC, PP, POM, and PEEK. The constant full-width-at-half-maximum of $(21.8 \pm 0.5) \mu \mathrm{m}$ is attributed to edge scattering and characterizes the $\mathrm{x}$-ray beam width. The edge scattering even shows a preferential orientation as indicated by the inset. The orientation is color-coded according to the color wheel inset. The gray area indicates the width of the cantilever, while the gradient of the gray color indicates the thickness. 


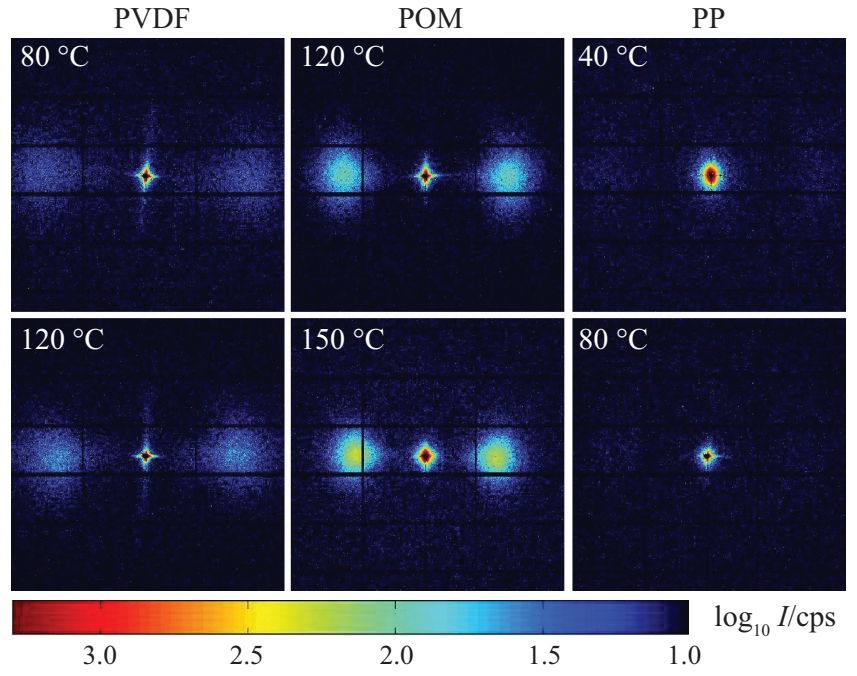

FIG. 5. The SAXS pattern at the center of PVDF, POM, and PP secondgeneration $\mu \mathrm{C}$ exhibit characteristic features, which become closer for higher mold temperatures. This means the observed nanostructures increase with the mold temperature.

cantilever rim. The thickness of the skin layer is expected to be in the same range or even smaller than the beam width and thus cannot be determined with this direct scanning SAXS approach, because the observed scattering effect overshadows the skin region.

In order to demonstrate the anisotropy of the $\mu \mathrm{C}, \mathrm{SAXS}$ patterns acquired within the center of the cantilevers were examined. Fig. 5 contains such background-corrected patterns for PVDF, POM, and PP $\mu \mathrm{C}$ fabricated using the mold temperatures indicated. Besides the central SAXS pattern around the direct-beam stop, one finds two diffraction spots in flow (horizontal) direction, which are more prominent for POM than for PVDF and PP. For PP, these spots are hardly visible, indicating low degree of crystallinity. The distance of the spots from the beam stop is material dependent and decreases with the mold temperature. This means that by increasing the mold temperature, larger nanostructures are formed.

We believe that the related periodicities are crystalline lamellae in the injection-molded polymers. The repeat spacing of the crystalline lamellae of semi-crystalline polymers produces these long-period SAXS patterns. The radial integration of the scattering patterns allows characterization of the lamellar periodicity through extraction of three empirical parameters, i.e., peak intensity $I_{\text {peak }}$, peak position $q_{\text {peak }}$, and

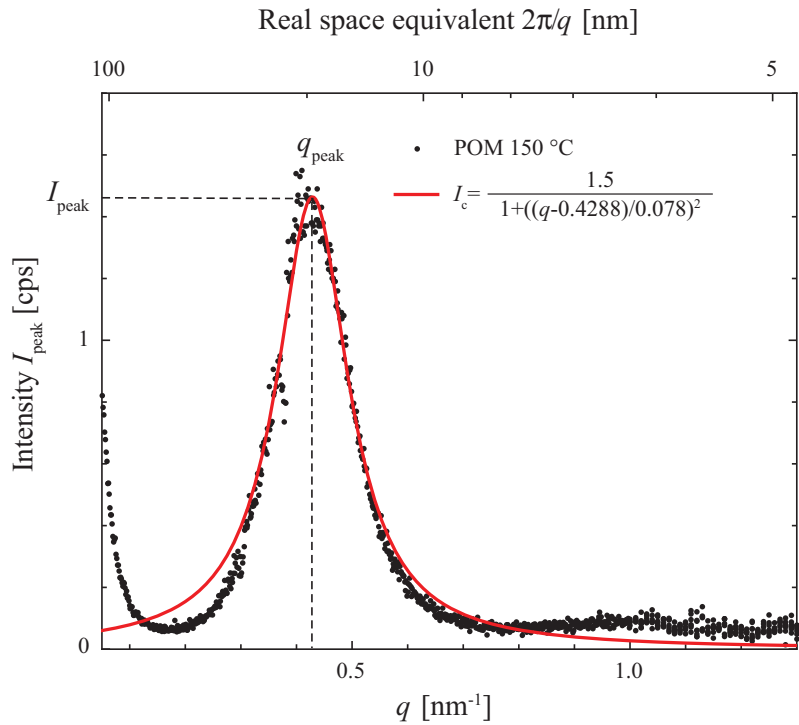

FIG. 6. The spots of the SAXS pattern shown in Fig. 5 are quantified using the peak intensity $I_{\text {peak }}$, the $q$-value at the peak $q_{\text {peak }}$, and the full-width-athalf-maximum FWHM $_{\text {peak }}$ of the spot derived from a fit to a Lorentzian (cf. Table I). The graph shows this procedure exemplarily for POM using the mold temperature of $150{ }^{\circ} \mathrm{C}$.

the full-width-at-half-maximum FWHM $_{\text {peak }}$ by fitting the peaks with a Lorentzian (cf. Fig. 6). Before fitting, the $q$-plots were background corrected with a correction function $I_{\text {corr }} \sim q^{-n}$. The correction exponent $n$ was chosen such that the baseline of the investigated peak was flat (cf. Fig. 6). The related $n$ was different for PVDF, POM, and PP and depended on the selected mold temperatures. The derived values of $n$ are compiled in Table I. After this background correction, the peaks in the $q$-plots can be reasonably fitted using a Lorentzian as the red-colored curve exemplarily demonstrates for a POM $\mu \mathrm{C}$ fabricated with a mold temperature of $150{ }^{\circ} \mathrm{C}$. Table I lists the fitted parameters for PVDF, POM, and PP at the selected mold temperatures. The decrease of $q_{\text {peak }}$ with mold temperature is the most striking feature. The higher mold temperature results in larger lamellar crystals.

Fig. 7 shows the variation of $I_{\text {peak }}, q_{\text {peak }}$, and the $\mathrm{FWHM}_{\text {peak }}$ across selected representative cantilevers. In the central part (constant thickness, cf. cantilever width given in gray), $I_{\text {peak }}$ forms a plateau with approximately constant height. It decreases with reducing cantilever thickness (cf. gradient in gray color). The FWHM peak and $q_{\text {peak }}$ show only negligible variations even when the $\mu \mathrm{C}$-thickness diminishes. The

TABLE I. Nanostructure characterization of micro-cantilevers injection-molded at different mold temperatures. Mean values and related standard deviations of the three Lorentzian fitted values and the degree of anisotropy of the central region of the cantilever.

\begin{tabular}{|c|c|c|c|c|c|c|}
\hline \multirow{2}{*}{$\begin{array}{l}\text { Material } \\
\text { Mold temperature }\left({ }^{\circ} \mathrm{C}\right)\end{array}$} & \multicolumn{2}{|c|}{ PVDF } & \multicolumn{2}{|c|}{ POM } & \multicolumn{2}{|c|}{ PP } \\
\hline & 80 & 120 & 120 & 150 & 40 & 80 \\
\hline$I_{\text {peak }}($ counts per second $)$ & $0.409 \pm 0.002$ & $0.776 \pm 0.046$ & $1.740 \pm 0.008$ & $1.478 \pm 0.014$ & $0.0568 \pm 0.0004$ & $0.353 \pm 0.005$ \\
\hline$q_{\text {peak }}\left(\mathrm{nm}^{-1}\right)$ & $0.6439 \pm 0.0010$ & $0.5591 \pm 0.0006$ & $0.5066 \pm 0.0005$ & $0.4287 \pm 0.0007$ & $0.6674 \pm 0.0027$ & $0.5485 \pm 0.0156$ \\
\hline $\mathrm{FWHM}_{\text {peak }}\left(\mathrm{nm}^{-1}\right)$ & $0.1991 \pm 0.0028$ & $0.1502 \pm 0.0013$ & $0.1013 \pm 0.0010$ & $0.0785 \pm 0.0015$ & $0.2203 \pm 0.0110$ & $0.2743 \pm 0.0389$ \\
\hline Correction exponent $(n)$ & 0.90 & 0.82 & 0.75 & 0.95 & 0.75 & 0.45 \\
\hline Degree of anisotropy & $0.717 \pm 0.005$ & $0.893 \pm 0.001$ & $0.953 \pm 0.002$ & $0.940 \pm 0.001$ & $0.440 \pm 0.019$ & $0.207 \pm 0.009$ \\
\hline
\end{tabular}




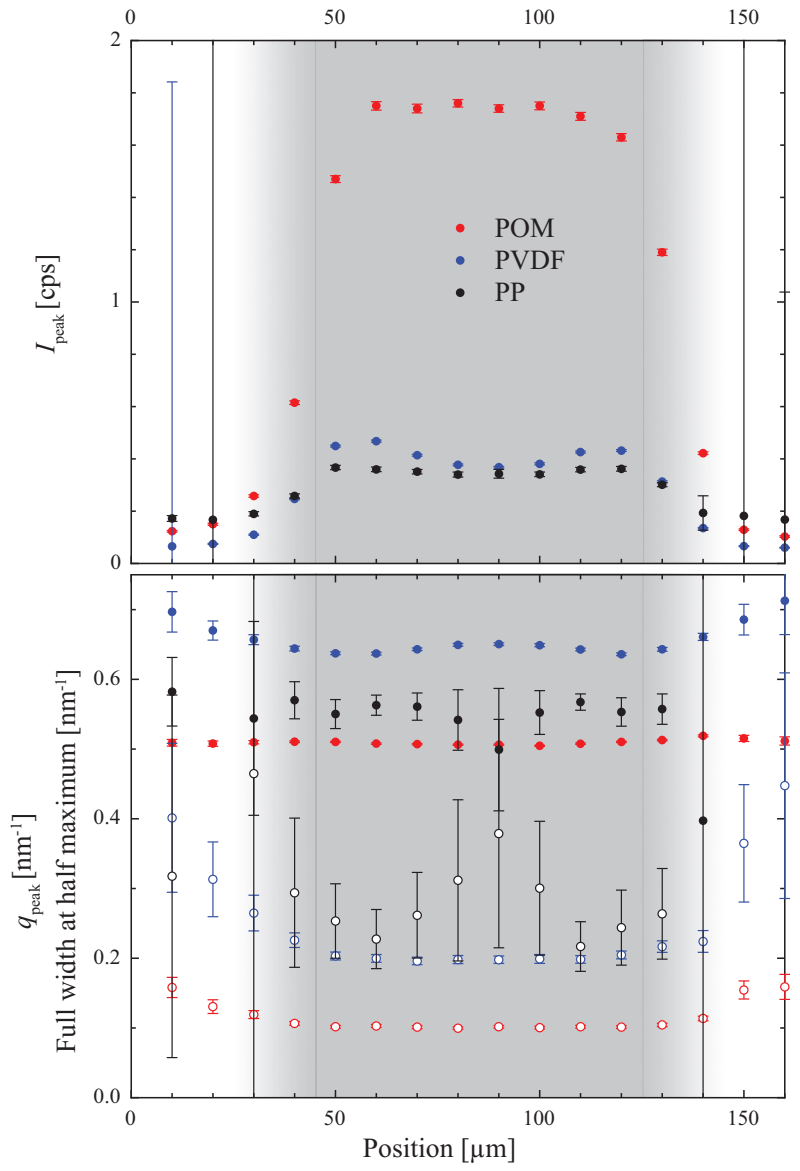

FIG. 7. The spatially resolved SAXS pattern (16 points across the width of the second-generation $\mu \mathrm{C}$ ) demonstrates the homogeneity of the POM, PVDF, and PP $\mu \mathrm{C}$ using the three fitted parameters (cf. Fig. 6).

relatively low scattering intensity for PP leads to large error bars. The low intensity variations in the central part of the $\mu \mathrm{C}$ indicate a homogeneous semi-crystalline structure.

The anisotropy of the $\mu \mathrm{C}$ related to the superstructures discussed above can be derived from the azimuthal intensity distribution. Fig. 8 displays such a plot for a POM $\mu \mathrm{C}$ fabricated at the mold temperature of $150{ }^{\circ} \mathrm{C}$. The diagram contains the mean scattered intensity in the $q$-range between 0.35 and $0.51 \mathrm{~nm}^{-1}$ values, averaged over six frames of $0.150 \mathrm{~s}$ exposure time each, as a function of the azimuthal angular position. The error bars correspond to the standard deviation between the frames. The air scattering signal was subtracted from each frame. The opposing segments were

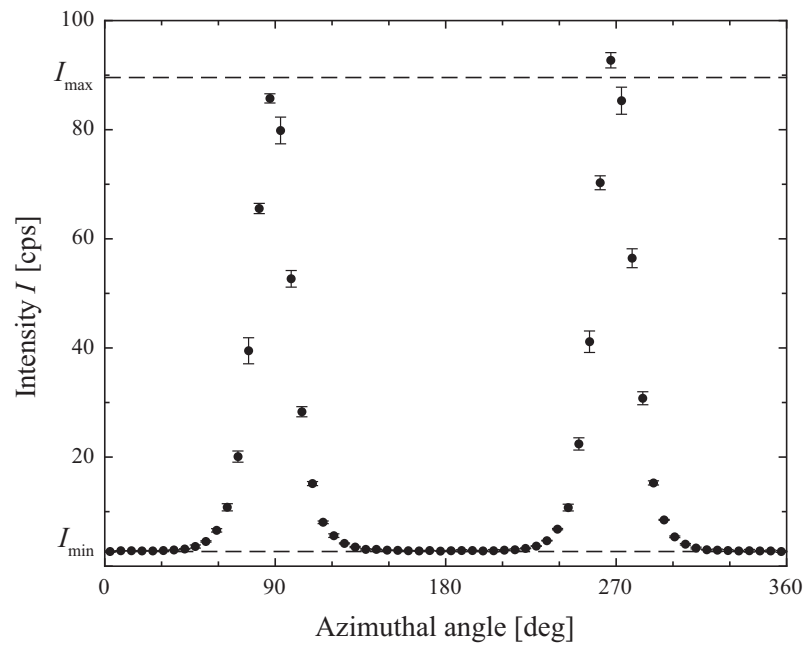

FIG. 8. The azimuthal plot ( $q$-range of $0.35-0.51 \mathrm{~nm}^{-1}$ ) of the mean scattered intensity of second-generation POM $\mu \mathrm{C}$ injection molded with a mold temperature $150^{\circ} \mathrm{C}$ elucidates the orientation of the related nanostructures. The degree of orientation is determined by means of $I_{\max }$ and $I_{\min }$.

averaged to gain the minimal and maximal intensities, $I_{\min }$ and $I_{\max }$ (cf. dashed lines in Fig. 8). The degree of anisotropy is defined as $\left(I_{\max }-I_{\min }\right) /\left(I_{\max }+I_{\min }\right)$. A strong orientation of the semi-crystalline lamellae stacks within the $\mu \mathrm{C}$ is observed for the three semi-crystalline polymers. The degree of anisotropy increases with mold temperature as summarized in Table I. Higher mold temperatures prevent early freezing. Therefore, the development of larger and welloriented nanostructures is enhanced. There is, however, no significant dependence on the injection speed as verified by the data shown in Table II. Both the degree of anisotropy and the $q_{\text {peak }}$-values of the three semi-crystalline polymers do not change for the different injection speeds.

\section{CONCLUSIONS}

Synchrotron radiation-based $\mathrm{x}$-ray scattering provides a wealth of information to quantitatively characterize injection-molded polymer microstructures. Contrary to the established skin-core morphology models with zones of different crystallinity, ${ }^{8,15}$ the spatially resolved SAXS and WAXS data elucidate that the $\mu \mathrm{Cs}$ are homogeneous in the scanning directions perpendicular to the beam. Their crystalline structure, however, exhibits a strong anisotropy. Both crystallinity and anisotropy can be controlled by changing the mold temperature but not the injection speed.

TABLE II. Nanostructure characterization of micro-cantilevers injection-molded with different injection speeds. Mean values and related standard deviations of the three Lorentzian fitted values and the degree of anisotropy of the central region of the cantilever.

\begin{tabular}{|c|c|c|c|c|c|c|c|}
\hline \multirow{2}{*}{$\begin{array}{l}\text { Material } \\
\text { Injection speed }\left(\mathrm{cm}^{3} / \mathrm{s}\right)\end{array}$} & \multicolumn{2}{|c|}{ PVDF } & \multicolumn{2}{|c|}{ POM } & \multicolumn{3}{|c|}{ PP } \\
\hline & 10 & 20 & 10 & 20 & 5 & 10 & 20 \\
\hline$I_{\text {peak }}($ counts per second) & $2.000 \pm 0.0004$ & $0.218 \pm 0.0004$ & $2.846 \pm 0.0144$ & $1.474 \pm 0.0036$ & $0.163 \pm 0.0008$ & $0.168 \pm 0.0008$ & $0.155 \pm 0.0008$ \\
\hline$q_{\text {peak }}\left(\mathrm{nm}^{-1}\right)$ & $0.562 \pm 0.0005$ & $0.558 \pm 0.0005$ & $0.512 \pm 0.0005$ & $0.513 \pm 0.0002$ & $0.545 \pm 0.0008$ & $0.549 \pm 0.0008$ & $0.555 \pm 0.0009$ \\
\hline $\mathrm{FWHM}_{\text {peak }}\left(\mathrm{nm}^{-1}\right)$ & $0.153 \pm 0.0015$ & $0.151 \pm 0.0014$ & $0.101 \pm 0.0009$ & $0.097 \pm 0.0004$ & $0.150 \pm 0.0019$ & $0.146 \pm 0.0019$ & $0.144 \pm 0.0019$ \\
\hline Correction exponent $(n)$ & 1.3 & 1.3 & 0.6 & 0.85 & 0.8 & 0.8 & 0.8 \\
\hline Degree of anisotropy & $0.950 \pm 0.003$ & $0.950 \pm 0.004$ & $0.950 \pm 0.004$ & $0.980 \pm 0.002$ & $0.850 \pm 0.006$ & $0.870 \pm 0.006$ & $0.860 \pm 0.004$ \\
\hline
\end{tabular}




\section{ACKNOWLEDGMENTS}

This research activity was funded by the Swiss Nanoscience Institute (SNI) through the applied research project DICANS, a collaborative initiative between the Biomaterials Science Center (BMC) of the University of Basel, the Paul Scherrer Institut (PSI), the University of Applied Sciences and Arts Northwestern Switzerland (FHNW), and Concentris GmbH. The authors thank O. Häfeli (FHNW Windisch) for the injection molding, K. Jefimovs (EMPA Dübendorf) and A. Stumpp (FHNW Windisch) for laser micro-machining of the mold, as well as X. Donath (PSI, Villigen) for support at the beamline. Experiments were performed on the cSAXS beamline at the Swiss Light Source, Paul Scherrer Institut, Villigen, Switzerland.

${ }^{1}$ L. Sawyer, D. Grubb, and G. Meyers, Polymer Microscopy (Springer Science, New York, 2008), Vol. 2.

${ }^{2}$ J.-W. Housmans, M. Gahleitner, W. M. P. Gerrit, and H. Meijer, Polymer 50, 2304 (2009).
${ }^{3}$ W. Michaeli and M. Bussmann, Shear-Induced Morphology Prediction in Injection Moulded Semi Crystalline Thermoplastics (2005).

${ }^{4}$ H. Mavridis, A. Hrymak, and J. Vlachopoulos, Polym. Eng. Sci. 26, 449 (1986).

${ }^{5}$ M. Gahleitner, J. Wolfschwenger, C. Bachner, K. Bernreitner, and W. Neiß1, J. Appl. Polym. Sci. 61, 649 (1996).

${ }^{6}$ M. Gahleitner, C. Bachner, E. Ratajski, G. Rohaczek, and W. Neiß1, J. Appl. Polym. Sci. 73, 2507 (1999).

${ }^{7}$ Z. Tadmor, J. Appl. Polym. Sci. 18, 1753 (1974).

${ }^{8}$ M. Kantz, H. Newman, and F. Stigale, J. Appl. Polym. Sci. 16, 1249 (1972).

${ }^{9}$ H. Ito, Y. Yagisawa, T. Saito, T. Yashuhara, T. Kikutani, and Y. Yamagiwa, Theor. Appl. Mech. Jpn. 54, 263 (2005).

${ }^{10}$ O. Bunk, M. Bech, T. Jensen, R. Feidenhans'1, T. Binderup, A. Menzel, and F. Pfeiffer, New J. Phys. 11, 123016 (2009).

${ }^{11}$ Y. D. Wang and M. Cakmak, Polymer 42, 3731 (2001).

${ }^{12}$ Y. D. Wang and M. Cakmak, Polymer 42, 4233 (2001).

${ }^{13}$ P. Urwyler, O. Häfeli, H. Schift, J. Gobrecht, F. Battiston, and B. Müller, Procedia Eng. 5, 347 (2010).

${ }^{14}$ M. C. Garcia-Gutierrez, A. Nogales, J. J. Hernandez, D. R. Rueda, and T. A. Ezquerra, Opt. Pura Apl. 40, 195 (2007).

${ }^{15}$ P.-W. Zhu and G. Edward, Macromol. Mater. Eng. 288, 304 (2003). 